\title{
Total Pressure Loss Mechanism of Centrifugal Compressors
}

\author{
Xiaoyang Gong ${ }^{1} \&$ Rui Chen ${ }^{1}$ \\ ${ }^{1}$ Department of Automotive and Aeronautics Engineering, Loughborough University, UK \\ Correspondence: Xiaoyang Gong, Department of Automotive and Aeronautics Engineering, Loughborough \\ University, UK. E-mail: gxy2013@hotmail.com
}

Received: September 10, 2014 Accepted: September 22, 2014 Online Published: October 17, 2014

doi:10.5539/mer.v4n2p45

URL: http://dx.doi.org/10.5539/mer.v4n2p45

\begin{abstract}
To develop the turbocharger matching system, a compressor model and turbine model need to be developed independently and coupled.

This paper describes the construction of the centrifugal compressor model and its validation with the experimental data. The compressor model in this paper uses One-dimensional (1D) thermo-fluid equations to analyse the compressor side of a turbocharger. Under a specified set of turbocharger geometry, atmospheric conditions, rotational speed, and fluid mass flow rate, the model can calculates the static and total temperatures, velocities, static and total pressures, pressure losses, and isentropic efficiencies for each compressor component. Instead of using lumped loss parameters, the compressor model includes established loss models found in the open literature. Not only in the impeller, the losses in the diffuser and the volute are modelled. With the model, it is possible for a parametric study on the effect of each loss mechanism on the performance, and which can aid the designer in justifying design decisions minimising the magnitude of the losses and thus positively influence the overall performance. The compressor model can also be applied to investigate the two stage Turbocharging or Variable Geometry Turbocharger (VGT) in the future.
\end{abstract}

Keywords: centrifugal compressor, total pressure losses, mechanism

\section{Nomenclature}

$\begin{array}{ll}\text { A } & \text { Reference flow area } \\ \mathrm{a} & \text { Velocity of sound } \\ \mathrm{b} & \text { Width } \\ \mathrm{C} & \text { Fluid absolute velocity } \\ \mathrm{C}_{\mathrm{f}} & \text { Surface friction loss coefficient } \\ \mathrm{D} & \text { Diameter } \\ \mathrm{r} & \text { Radius } \\ \mathrm{Re} & \text { Reynolds number } \\ \mathrm{T} & \text { Static Temperature } \\ \mathrm{P} & \text { Static Pressure } \\ \rho & \text { Density } \\ \mathrm{M} & \text { Mach number } \\ \mathrm{U} & \text { Tangential velocity } \\ \mathrm{W} & \text { Relative velocity } \\ \alpha & \text { Absolute flow angle } \\ \beta & \text { Relative flow angle } \\ l_{i} & \text { The mean channel length of impeller } \\ l_{d} & \text { The mean channel length of diffuser } \\ D_{i} & \text { The mean hydraulic channel diameter of impeller }\end{array}$


$D_{d} \quad$ The mean hydraulic channel diameter of diffuser

$Z_{c} \quad$ The impeller blade number

$\mathrm{C}_{\mathrm{p}} \quad$ Specific heat capacity at constant pressure

$\mathrm{C}_{\mathrm{v}} \quad$ Specific heat capacity at constant volume

f Friction factor

$\gamma \quad$ Ratio of specific heats. $\gamma=\frac{C_{p}}{C_{v}}$

$\sigma \quad$ Slip factor

h Specific enthalpy

$\eta_{c s} \quad$ Compressor isentropic efficiency

PR Pressure ratio of compressor

$\omega \quad$ Pressure Loss

\section{Subscripts}

$\begin{array}{ll}\mathrm{u} & \text { Tangential fluid velocity } \\ \mathrm{r} & \text { Axial fluid velocity } \\ \mathrm{m} & \text { Meridional coordinate direction } \\ \mathrm{h} & \text { Hub } \\ \mathrm{t} & \text { Tip } \\ \mathrm{th} & \text { Throat parameter } \\ 0 & \text { Stagnation temperature or Pressure } \\ 1 & \text { Impeller blade inlet } \\ 2 & \text { Impeller exit } \\ 3 & \text { Vaneless diffuser exit } \\ 4 & \text { Vaned diffuser exit } \\ 5 & \text { Volute exit }\end{array}$

\section{Introduction}

Worldwide regulators are setting stringent fuel economy and emissions standards that are challenging auto manufacturers to create less emission, more fuel efficient passenger cars. As vehicle manufacturers weigh up the cost-benefits of reaching these incoming targets, analysts agree that one technology in particular - turbocharging will play a central enabling role (Watson \& Janota, 1982). While turbodiesels will remain the dominant powertrain, stricter EU regulations on emissions and strong growth in boosted gasoline engines mean that by 2020 , around $85 \%$ of all new light vehicles in Europe will be turbocharged, in line with demand for smaller, better performing engines (Baines, 2005).

The prediction of compressor performance in the early design stage of a turbocharger is critical and helps to ensure that the range and matching of the constituent components (impeller, diffuser and volute) is satisfactory. Performance maps provided by the turbocharger manufacturers are usually limited to a reduced number of rotational speeds, pressure ratios and mass flow rates. Extrapolation of map data is commonly required and there is no physical meaning. A comprehensive prediction technique, which enables the designer to examine the performance of all the individual components within the compressor at the initial design stage, would result in a device designed to its optimum specification (Watson \& Janota, 1982). The major gain from establishing a more accurate prediction technique would enable fewer uncertainties in both design time and production costs and would allow existing designs of centrifugal compressors to be improved. Despite impressive progress in computational fluid dynamics procedures, one-dimensional prediction techniques continue to be the most accurate and the most practical method of predicting the performance of a stage or a component of a stage (Watson \& Janota, 1982)

During the past several decades, the one-dimensional prediction techniques and the empirical loss correlation method has been persistently developed, which is well documented in standard textbooks such as those by Dixon (1978), Whitfield and Baines (1990), Yahya (1983). In a number of open literature sources such as Watson and Janota (1982) and Japikse and Baines (1997), losses are typically lumped together as a slip factor and/or loss 
coefficient. The downside of such a treatment is that effects of individual losses are not clearly characterized and thus their influence is poorly understood. Takagi, Kobayashi, Miyashiro, and Morimoto (1980) formulated loss models for leakage flow and disc friction based on their measurements of centrifugal pumps at three different specific speeds. Thanapandi and Prasad (1990) surveyed number of available loss correlations and found a satisfactory set of models for almost the full range of operating conditions of low specific speed submersible pumps. Recently Aungier (1995) presented a modular performance prediction procedure which has been validated against about one hundred different stages with a wide range of flow coefficients and pressure ratios up to about 3.5.

This paper aims to bring all potential losses in each component of compressor together to reveal the full total pressure loss mechanism in centrifugal compressor. The model in this paper is based on the thermodynamics, i.e. where energy transfer is being taken into consideration including the losses is determined from the compressor geometry. With the model presented here, we can predict the compressor performance curves such as outlet pressure, isentropic efficiency and different losses in each component. The losses can also be analysed individual to produce improved thermodynamic compressor efficiency.

\section{Compressor Model}

\subsection{Impeller Model}

The most critical and geometrically complex component of the compressor is the impeller. It is the only rotating component and as such, transfers energy to the air resulting in an increase in pressure, velocity and enthalpy. A key geometric feature of the impeller is the inlet area, $\mathrm{A}_{1}$, which can be found from the relationship:

$$
A_{1}=\frac{\pi}{4}\left(D^{2}{ }_{t 1}-D^{2}{ }_{h 1}\right)
$$

where $D_{t 1}$ and $D_{h 1}$ are the diameter at the tip and the hub section of the impeller respectively.

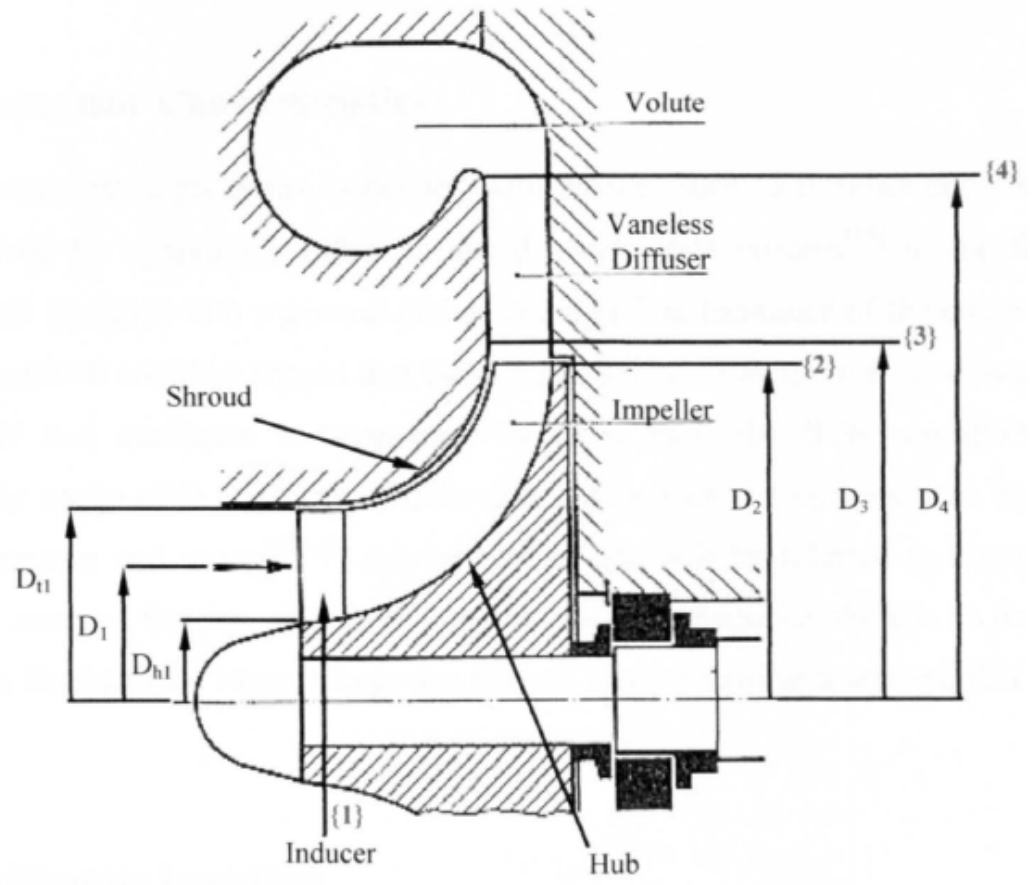

Figure 1. Meridional view of centrifugal compressor (Japikse \& Baines, 1997)

The non-dimensional losses $(\bar{\omega})$ of the components are computed as functions of the geometry, velocity, temperature, and pressure. The impeller is the only rotating component in the compressor so the losses of impeller are converted into a total relative pressure loss described as (Aungier, 1995):

$$
\Delta \mathrm{P}_{\mathrm{tr}}=f_{c}\left(\mathrm{P}_{\mathrm{tr} 1}-\mathrm{P}_{1}\right) \sum_{i} \bar{\omega}_{i}
$$


where $\mathrm{P}_{\operatorname{tr} 1}$ and $\mathrm{P}_{1}$ are the total relative pressure and static pressure at impeller exit respectively, $f_{c}$ is a correction factor and can be calculated as

$$
f_{c}=\frac{\rho_{\operatorname{tr} 2} T_{\operatorname{tr} 2}}{\rho_{\operatorname{tr} 1} T_{\operatorname{tr} 1}}
$$

where $\rho_{\text {tr }}$ is the total relative density, and $\mathrm{T}_{\text {tr }}$ is the total relative temperature.

The total relative pressure $P_{t r}$ and total relative temperature $T_{t r}$ can be calculated as (Aungier, 1995)

$$
\begin{gathered}
\mathrm{P}_{\mathrm{tr}}=\mathrm{P}\left[1+\left(\frac{\gamma-1}{2}\right) \mathrm{M}_{\mathrm{r}}^{2}\right]^{\frac{\gamma}{\gamma-1}} \\
\mathrm{~T}_{\mathrm{tr}}=\mathrm{T}\left[1+\left(\frac{\gamma-1}{2}\right) \mathrm{M}_{\mathrm{r}}^{2}\right]
\end{gathered}
$$

where $M_{r}$ is the relative Mach number, which can be mathematically described as

$$
\mathrm{M}_{\mathrm{r}}=\frac{\mathrm{w}}{\mathrm{a}}
$$

where $\mathrm{W}$ is the relative velocity and a is the velocity of sound.

To calculate the non-dimensional loss parameters in the impeller, isentropic conditions are determined first. The losses are then applied to the isentropic conditions to calculate the actual conditions.

The non-isentropic parameters are evaluated with the assumption that rothalpy is conserved. Rothalpy is defined as the difference between the total enthalpy and angular momentum (Aungier, 1995). For the stationary component, conservation of energy was applied. Similarly, the conservation of rothalpy is used for the rotating impeller. Conservation of rothalpy requires that the isentropic relative total temperature is equal the actual relative total temperature.

$$
\mathrm{T}_{\text {trideal }}=\mathrm{T}_{\text {tr,actual }}
$$

The actual total relative pressure at the impeller exit is the difference between the isentropic total relative pressure and the total relative pressure loss, mathematically described as

where $\mathrm{P}_{\text {tr2,ideal }}$ can be calculated as

$$
\mathrm{P}_{\text {tr2,actual }}=\mathrm{P}_{\text {tr2,ideal }}-\Delta \mathrm{P}_{\text {tr }}
$$

$$
\mathrm{P}_{\text {tr2,ideal }}=\mathrm{P}_{2, \text { ideal }}\left[1+\left(\frac{\gamma-1}{2}\right) M_{r 2}\right]^{\frac{\gamma}{\gamma-1}}
$$

Nine losses that affect the impeller performance have been addressed in the impeller model. These are:

1) Shock loss - is estimated by assuming that the loss is entirely generated by a normal shock at the inlet (Whitfield \& Baines, 1990). The shock loss can be modelled as

$$
\bar{\omega}_{s h}=1-\left(\frac{W_{T h}}{W_{1}}\right)^{2}-\left(\frac{2}{(\gamma-1) M_{r 1}^{2}}\right)\left[\left(\frac{P_{T h}}{P_{1}}\right)^{\frac{\gamma-1}{\gamma}}-1\right]
$$

where $W_{T h}$ is the impeller throat relative velocity and which can be calculated as

$$
W_{T h}=\frac{\mathrm{C}_{\mathrm{rTh}}}{\sin \left(\beta_{T h}\right)}
$$

where $\mathrm{C}_{\mathrm{rTh}}$ is the throat radial velocity and $\beta_{T h}$ is the relative blade angle at the throat.

2) Incidence loss - accounts for the flow adjustment of the actual fluid flow to the blade angle at the inlet of the impeller (Aungier, 1995). The incidence loss can be modelled as

$$
\bar{\omega}_{\text {inc }}=0.8\left[1-\frac{C_{r 1}}{W_{1} \sin \left(\theta_{m 1}\right)}\right]^{2}+\left[\frac{t_{b 1} \cdot z}{2 \cdot \pi \cdot r_{1} \cdot \sin \left(\theta_{m 1}\right)}\right]^{2}
$$

where $t_{b 1}$ is the impeller inlet blade thickness and $\theta_{m 1}$ is the inlet mean blade angle.

3) Clearance Loss - Some of the flow exiting the impeller leaks through the clearance gaps and labyrinth seals to the lower pressure regions of the compressor, causing the clearance loss (Moussa, 1978). The clearance loss can be modelled as 


$$
\bar{\omega}_{c l}=\frac{2 \dot{m}_{c l} \Delta P_{c l}}{\dot{m} \rho_{1} W_{1}^{2}}
$$

$\Delta P_{c l}$ is the pressure difference across the gap and $\dot{m}_{c l}$ is the clearance gap leakage flow rate.

4) Skin friction loss - The loss generating from the fluid flowing over the surface, which is also known as wall friction loss (Aungier, 1995). The skin friction loss in impeller can be modelled as

$$
\begin{gathered}
\bar{\omega}_{s f}=4 C_{f}\left(\frac{\bar{W}}{W_{1}}\right)^{2} \frac{L_{B}}{D_{H}} \\
\bar{W}^{2}=\frac{\left(W_{1}{ }^{2}+W_{2}{ }^{2}\right)}{2}
\end{gathered}
$$

where $L_{B}$ is the blade mean camberline length, $D_{H}$ is the hydraulic diameter, $b_{2}$ is the impeller exit width, $\theta_{2}$ is the discharge blade angle and $R e_{d}$ is the Reynolds number.

5) Blade loading loss - accounts for the blade-to-blade pressure gradient that produces secondary flows that potentially lead to stall (Thanapandi \& Prasad, 1990). The Blade loading loss can be modelled as

$$
\bar{\omega}_{b l}=\frac{\left(\Delta W / W_{1}\right)^{2}}{24}
$$

where $\Delta W$ is the average blade velocity difference.

6) Hub to shroud loading loss - accounts for the pressure gradient in the hub-to-shroud direction. This pressure gradient produces secondary flows that can lead to stall (Oh, Yoon, \& Chung, 1997). The hub to shroud loading loss can be calculated as

$$
\bar{\omega}_{h s}=\frac{\left(\bar{k}_{m} \bar{b} \bar{W} / W_{1}\right)^{2}}{6}
$$

where $\bar{W}$ is the average relative velocity, $\bar{k}_{m}$ is the passage curvature, $\bar{b}$ is the average width.

7) Blockage loss - accounts for the mixing of the distorted meridional velocities (Oh, Yoon, \& Chung, 1997). The blockage loss in impeller can be calculated as

$$
\bar{\omega}_{\exp }=\left[\frac{(\lambda-1) C_{r 2}}{W_{1}}\right]^{2}
$$

where $\lambda$ is the tip distortion factor.

8) Mixing loss - accounts for the mixing of blade wake flows with the free stream flow (Aungier, 1995). The wake mixing loss can be calculated as

$$
\bar{\omega}_{\text {mix }}=\left[\frac{C_{r, w a k e}-C_{r, m i x}}{W_{1}}\right]^{2}
$$

where $C_{r, \text { wake }}$ is the radial velocity of the wake and $C_{r, \text { mix }}$ is the radial velocity of the mixed flow.

9) Supercritical Mach number loss - accounts for the shock wave losses produced by boundary layer separation when blade surface velocities reach supersonic conditions (Aungier, 1995). The supercritical Mach number loss can be calculated as

$$
\bar{\omega}_{s c m n}=0.4\left[\frac{\left(M_{r 1}-M_{c r}\right) W_{\max }}{W_{1}}\right]^{2}
$$

where $M_{c r}$ is the inlet critical Mach number.

The isentropic solution is used in conjunction with the loss models to evaluate the non-isentropic or actual conditions. The actual exit conditions can be used to determine the component mean thermodynamic efficiency and power consumption parameters.

\subsection{Diffuser Model}

\subsubsection{Vaneless Diffuser}

Centrifugal compressors are usually fitted with either a vaneless (annular) or a vaned diffuser. A considerable proportion of the fluid energy at the impeller tip is kinetic energy and its efficient transformation into static pressure is important. The vaneless diffuser is a simple annular channel in which the fluid loses velocity and gains 
static pressure. The capability of diffusion can be evaluated by the reduce degree of the velocities at the outlet and inlet of vaneless diffuser.

The non-dimensional loss parameter for the vaneless diffuser is converted into a total pressure loss (Aungier, 1995). The total pressure loss conversion can be mathematically described as

$$
\Delta P_{t 3}=\left(P_{t 2}-P_{2}\right) \sum_{i} \bar{\omega}_{i}
$$

where $P_{t 2}$ and $P_{2}$ are the total pressure and static pressure at impeller exit respectively.

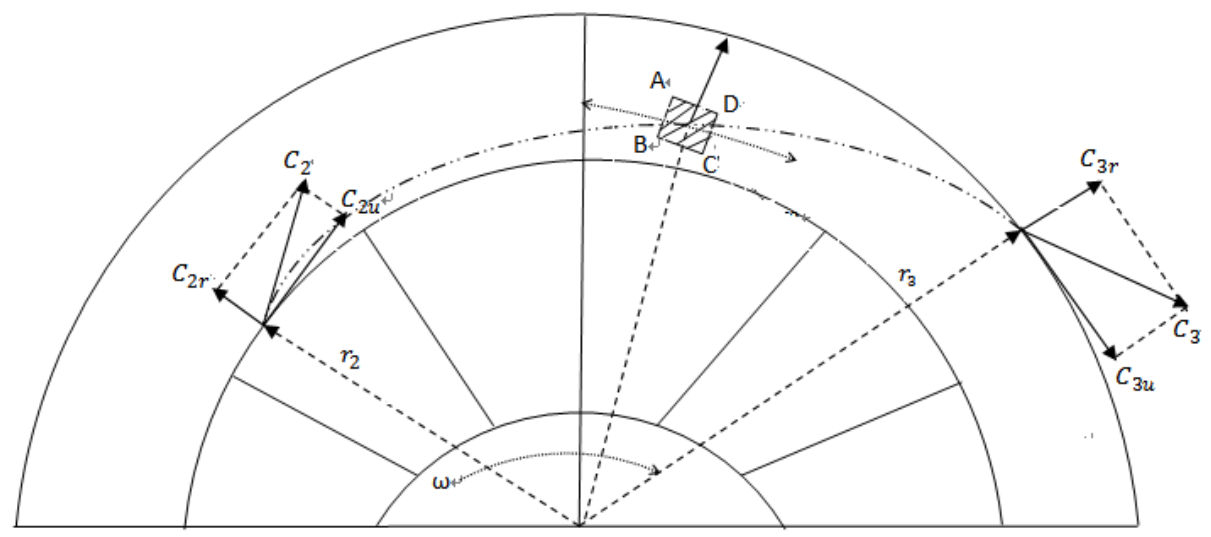

Figure 2. Flow inside the Vaneless diffuser

The vaneless diffuser inlet conditions have been calculated as equal to the impeller actual exit conditions. Two losses in the vaneless diffuser are modelled in this paper.

1) Skin friction loss - similar as the impeller, skin friction loss is the loss generating from the fluid flowing over the wall (Aungier, 1995). The skin friction loss can be calculated as

$$
\bar{\omega}_{s f}=4 C_{f}\left(\frac{\bar{C}}{C_{2}}\right)^{2} \frac{\left(r_{3}-r_{2}\right)}{D_{\text {hyd }}}
$$

where $\bar{C}$ is the average velocity and it can be calculated as

$$
\bar{C}^{2}=\frac{\left(C^{2}{ }_{2}+C^{2}{ }^{2}\right)}{2}
$$

2) Diffusion loss - happens in the process of diffusion and is estimated using a correlation for momentum thickness using the diffusion ratio as the driving parameter (Johnston \& Dean, 1966). The diffusion loss can be calculated as

$$
\bar{\omega}_{d f}=-2(1-\mathrm{E})\left(\frac{C_{3, i d}-C_{2}}{\rho_{2} C_{2}}\right)
$$

where $\mathrm{E}$ is the diffusion efficiency.

The actual total pressure is the difference of the ideal total pressure and the total pressure loss.

$$
\mathrm{P}_{\mathrm{t} 3 \text {,actual }}=\mathrm{P}_{\mathrm{t} 3 \text {,ideal }}-\Delta \mathrm{P}_{\mathrm{t} 3}
$$

The actual total pressure is used to calculate the other actual exit conditions of the vaneless diffuser using the velocity diagrams, total temperature relationship to calculate the static temperature, the total pressure relationship to calculate the static pressure, the density, and the assumption that the total temperature is constant due to constant enthalpy. The actual exit conditions can be used to determine the component mean thermodynamic efficiency and power consumption parameters.

\subsubsection{Vaned Diffuser}

The overall rate of diffusion in the vaned diffuser is higher than that of the vaneless diffuser for the same radius ratio. The vaned diffuser is more preferable than vaneless one when more compact compressor designs are required. 
Similar to the vaneless diffuser, the non-dimensional loss parameter for the vaned diffuser is converted into a total pressure loss. The total pressure loss conversion can be mathematically described as

$$
\Delta P_{t 4}=\left(P_{t 3}-P_{3}\right) \sum_{i} \bar{\omega}_{i}
$$

where $P_{t 3}$ and $P_{3}$ are the total pressure and static pressure at vaneless diffuser exit respectively (Aungier, 1995).

The loss parameters are calculated using the inlet, throat, and isentropic exit conditions. The inlet conditions are equal to the actual exit conditions of the vaneless diffuser.

1) Skin friction loss - similar as the impeller, skin friction loss is the loss generating from the fluid flowing over the surface (Aungier, 1995). The skin friction loss can be calculated as

$$
\bar{\omega}_{s f}=4 C_{f}\left(\frac{\bar{C}}{C_{3}}\right)^{2} \frac{L_{B} / D_{H}}{\left(2 \delta / D_{H}\right)^{0.25}}
$$

where $\frac{2 \delta}{D_{H}}$ is the boundary layer approximation.

2) Incidence loss - similar as the impeller, the incidence loss occurs when the relative fluid flow angle of the fluid entering the vane deviates from the actual blade inlet angle (Aungier, 1995). The incidence loss can be calculated as

$$
\begin{gathered}
\bar{\omega}_{i n c}=0.8\left(\frac{C_{3}-C^{*} 3}{C_{3}}\right)^{2} ; \quad C_{3} \leq C_{3 s} \\
\bar{\omega}_{i n c}=0.8\left[\left(\left(\frac{C_{3}}{C_{3 s}}\right)^{2}-1\right) \frac{C^{2} T h}{C^{2}{ }_{3}}+\frac{C_{3 s}-C^{*}}{C^{2}{ }_{3 s}}\right] ; C_{3}>C_{3 s}
\end{gathered}
$$

where $C_{3 S}$ is the absolute stall velocity and $C_{3}^{*}$ is the velocity at the optimum incidence angle.

3) Blockage loss - similar as the impeller, blockage loss accounts for the mixing of the distorted meridional velocities (Oh, Yoon, \& Chung, 1997). The blockage loss can be calculated as

$$
\bar{\omega}_{e x}=\left[\frac{(\lambda-1) C_{r 4}}{C_{4}}\right]^{2}
$$

where $\lambda$ is the tip distortion factor.

4) Wake mixing loss - similar as the impeller, wake mixing loss accounts for the mixing of blade wake flows with the free stream flow (Aungier, 1995). The wake mixing loss can be calculated as

$$
\bar{\omega}_{\text {mix }}=\left[\frac{C_{r, w a k e}-C_{r, m i x}}{C_{3}}\right]^{2}
$$

where $C_{r, \text { wake }}$ is the radial velocity of the wake and $C_{r, \text { mix }}$ is the radial velocity of the mixed flow.

Similar as the impeller, the actual total pressure is the difference of the ideal total pressure and the total pressure loss.

\subsection{Volute Model}

$$
\mathrm{P}_{\mathrm{t} 4, \text { actual }}=\mathrm{P}_{\mathrm{t} 4 \text {,ideal }}-\Delta \mathrm{P}_{\mathrm{t} 4}
$$

The volute is the final component of the centrifugal compressor. Many of the volute designs have remained unchanged in decades and since there is only a small gain in pressure ratio in this component, the volute is generally treated as a simple means of directing the air flow and not as an additional component to improve performance. The non-dimensional loss parameter for the vaned diffuser is converted into a total pressure loss. The total pressure loss conversion can be mathematically described as

$$
\Delta P_{t 5}=\left(P_{t 4}-P_{4}\right) \sum_{i} \bar{\omega}_{i}
$$

where $P_{t 4}$ and $P_{4}$ are the total pressure and static pressure at vaned diffuser exit respectively (Aungier, 1995). The inlet conditions of the volute are equal to the exit conditions of the actual vaned diffuser.

There are three losses described in the volute.

1) Meridional velocity loss - it assumes that the head associated with the meridional velocity at the volute inlet is lost (Weber \& Koronowski, 1986).

$$
\bar{\omega}_{m}=\left(\frac{C_{4 r}}{C_{4}}\right)^{2}
$$


2) Tangential velocity loss - occurs if the tangential velocity decreases from the volute inlet to the exit and then the flow diffuses (Weber \& Koronowski, 1986).

$$
\begin{aligned}
& \bar{\omega}_{U}=\frac{1}{2} \frac{r_{4} C^{2}{ }_{4 u}}{r_{5} C^{2}{ }_{4}}\left[1-\frac{1}{S P^{2}}\right] ;(\mathrm{SP} \geq 1) \\
& \bar{\omega}_{U}=\frac{r_{4} C^{2}{ }_{4 u}}{r_{5} C^{2}{ }_{4}}\left[1-\frac{1}{S P}\right]^{2} ;(\mathrm{SP}<1)
\end{aligned}
$$

where SP is the volute sizing parameter.

3) Skin friction loss - similar as the impeller, skin friction loss is the loss generating from the fluid flowing over the surface (Aungier, 1995).

$$
\bar{\omega}_{s f}=4 C_{f}\left(\frac{C_{5}}{C_{4}}\right)^{2} \frac{L}{D_{H}}
$$

where $\mathrm{L}$ is the mean stream line path length of volute and $D_{H}$ is the hydraulic diameter of volute.

Similar as the impeller, the actual total pressure is the difference of the ideal total pressure and the total pressure loss.

\subsection{Compressor Model}

$$
\mathrm{P}_{\mathrm{t} 5, \text { actual }}=\mathrm{P}_{\mathrm{t} \text {, ideal }}-\Delta \mathrm{P}_{\mathrm{t} 5}
$$

\subsubsection{The Model}

In this paper, the compressor is separated into its individual components for modelling purposes. Each component inlet and exit characteristics are defined as stations. The compressor can be described using five stations. Station one is at the impeller inlet while the impeller exit and vaneless diffuser inlet is station two. The conditions for the impeller exit and vaneless diffuser inlet are the same. Station three is at the vaneless diffuser exit or the vaned diffuser inlet. Again, the vaneless diffuser exit and vaned diffuser inlet have the same conditions. Station four is at the vaned diffuser exit or the volute inlet. Finally, station five is at the volute exit.

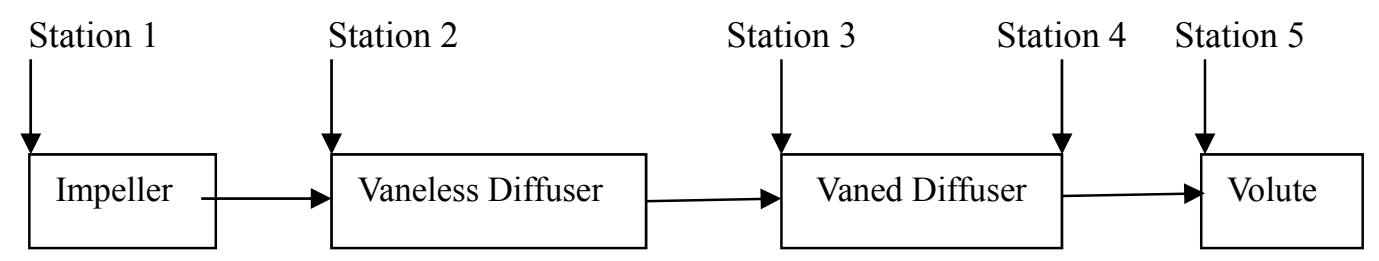

Figure 3. Compressor Component Division

For each component of compressor, a set of equations and associated variables were used to fully describe it. Variables which influence the component inlet are the inlet pressure, inlet temperature, and inlet velocities. Meanwhile, the exit pressure, exit temperature, and exit velocities influence the component exit. Finally, the isentropic efficiency of each component is calculated because it influences across the component. A set of algebraic equations will relate the input and output conditions. The equations were developed from the moment of momentum equation, steady flow energy equation, mass continuity equation, thermodynamic equation of state, and trigonometric relations from corresponding velocity vector diagrams at the mean stream line. 


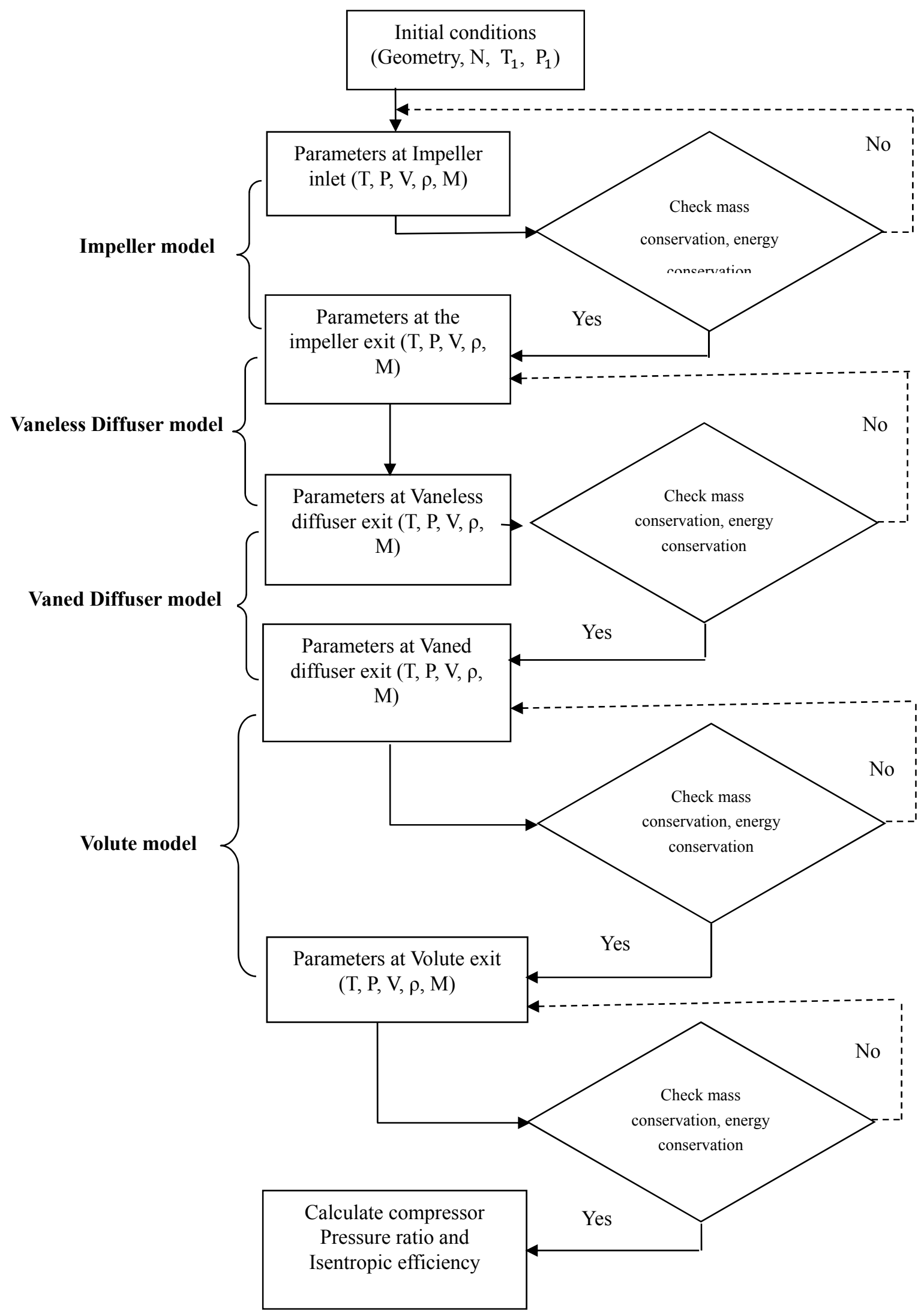

Figure 4. Flow Chart for the Compressor Performance Prediction 


\subsubsection{Power Consumption}

Compressor power consumption can be described using Euler's turbomachinery equation. Euler's equation is the combination of basic energy conservation and conservation of angular momentum which provides compressor power in terms of the tangential velocity (Whitfield \& Baines, 1990). The relationship is mathematically described as:

\subsubsection{Compressor Pressure Ratio}

$$
\mathrm{h}_{\mathrm{t}, \text { out }}-\mathrm{h}_{\mathrm{t}, \text { in }}=\mathrm{C}_{\mathrm{U}, \text { out }} \mathrm{U}_{\text {out }}-\mathrm{C}_{\mathrm{U}, \text { in }} \mathrm{U}_{\text {in }}
$$

The pressure ratio of each compressor component can be easily determined from the pressure at the exit and at the inlet.

$$
\mathrm{PR}=\frac{\mathrm{P}_{\text {out }}}{\mathrm{P}_{\text {in }}}
$$

\subsubsection{Compressor Isentropic Efficiency}

The compressor component mean isentropic thermodynamic efficiency is a function of compressor inlet and exit total (the sum of the static and dynamic condition) pressure and total temperature and is mathematically described as (Dixon, 1978):

$$
\eta=\frac{\left(\frac{P_{t, o u t}}{P_{t, i n}}\right)^{\frac{\gamma-1}{\gamma}}-1}{\left(\frac{T_{, o u t}}{T_{t, \text { in }}}\right)-1}
$$

The isentropic efficiency of each compressor component can be calculated from the equation above.

\section{Validation}

The proposed model was validated against experimental results which is found from the literature (Chapman, Keshavarz, \& Honegger, 2007). The geometry data of the centrifugal compressor was found in open literature and which is collected by the researchers at the National Gas Machinery Laboratory (NGML) on the VGT turbocharger, developed from an ET-18 compressor (Chapman et al., 2007).

Table 1. The operating conditions and geometry data of compressor (Chapman et al., 2007)

\begin{tabular}{llll}
\hline Parameter & Value & Parameter & Value \\
\hline Inlet Static Temperature ( K) & 283.84 & Impeller Inlet Hub Diameter (m) & 0.1106 \\
Inlet Static Pressure (KPa) & 96.53 & Impeller Exit Diameter (m) & 0.4572 \\
Rotational Speed (rpm) & $10000 \sim 14500$ & Impeller Exit Blade Angle $\left({ }^{\circ}\right)$ & 26.2377 \\
Impeller Inlet Tip Diameter (m) & 0.2870 & Vaned diffuser inlet diameter (m) & 0.5410 \\
Impeller Inlet Hub Diameter (m) & 0.1106 & Vaned diffuser exit diameter (m) & 0.6332 \\
Impeller Inlet Blade Thickness (m) & 0.0037 & Vaned diffuser exit Blade Angle $\left({ }^{\circ}\right)$ & 46.2720 \\
Impeller Blade inlet angle $\left({ }^{\circ}\right)$ & 45 & Volute inlet diameter (m) & 0.6332 \\
Impeller Throat Mean Blade Angle $\left({ }^{\circ}\right)$ & 49.0230 & Volute exit diameter (m) & 0.7620 \\
\hline
\end{tabular}

Figure 5 shows the comparison between the simulation results at three different rotational speeds with the experiment results presented in the literature (Chapman et al., 2007). The operating conditions of compressor and geometry data was the same as the experiment, which has been given in Table 1.

As shown in the figure, the simulation results shows quite a similar trend with the experiment results. However, the simulation results shows a little underestimated with the experiment results when the mass flow rate goes up. The reason for this might be due to some other losses (disc friction, choking, etc.), which are not taken account in this model. 


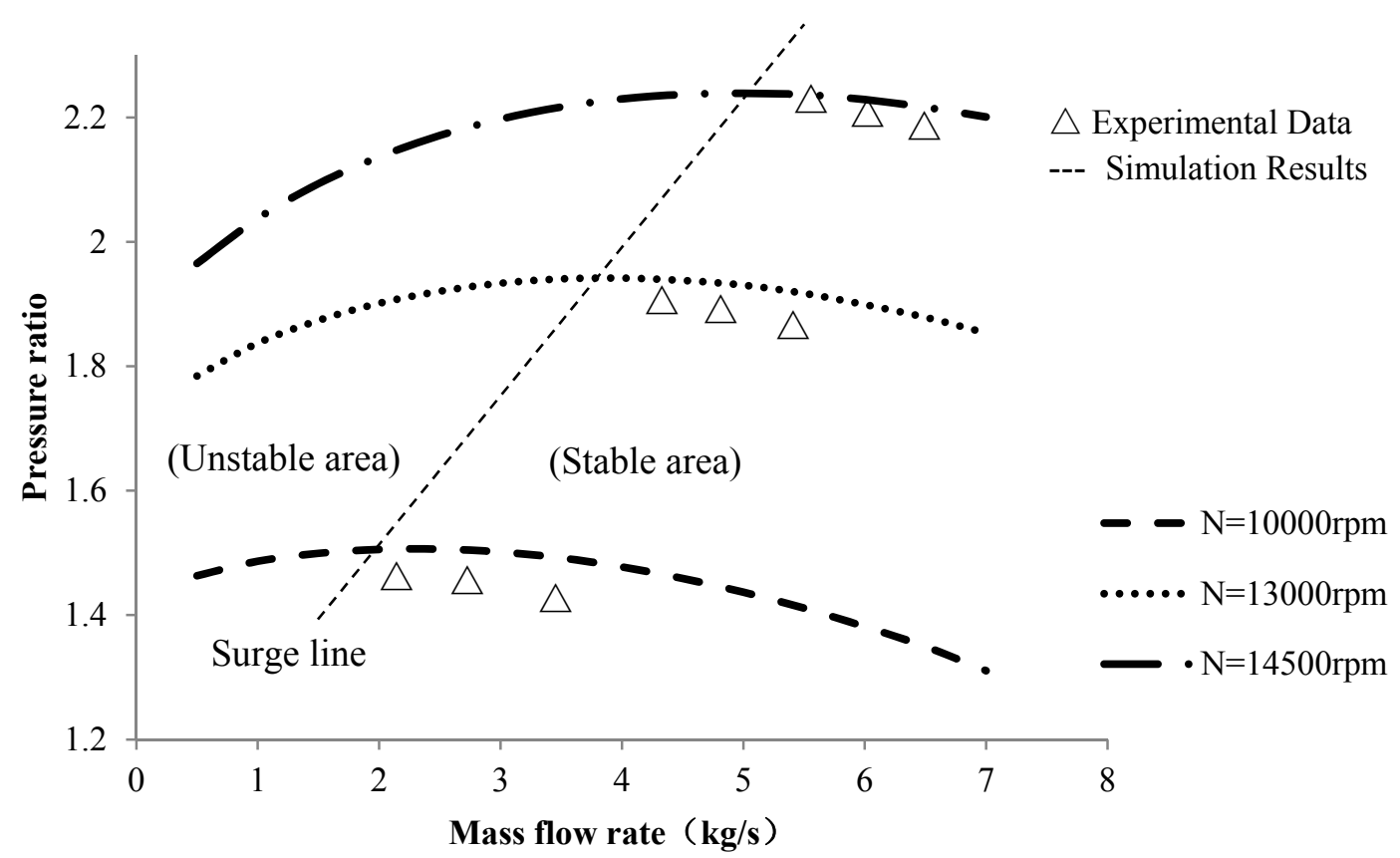

Figure 5. Compressor Pressure ratio at different rotational speed

\section{Discussion}

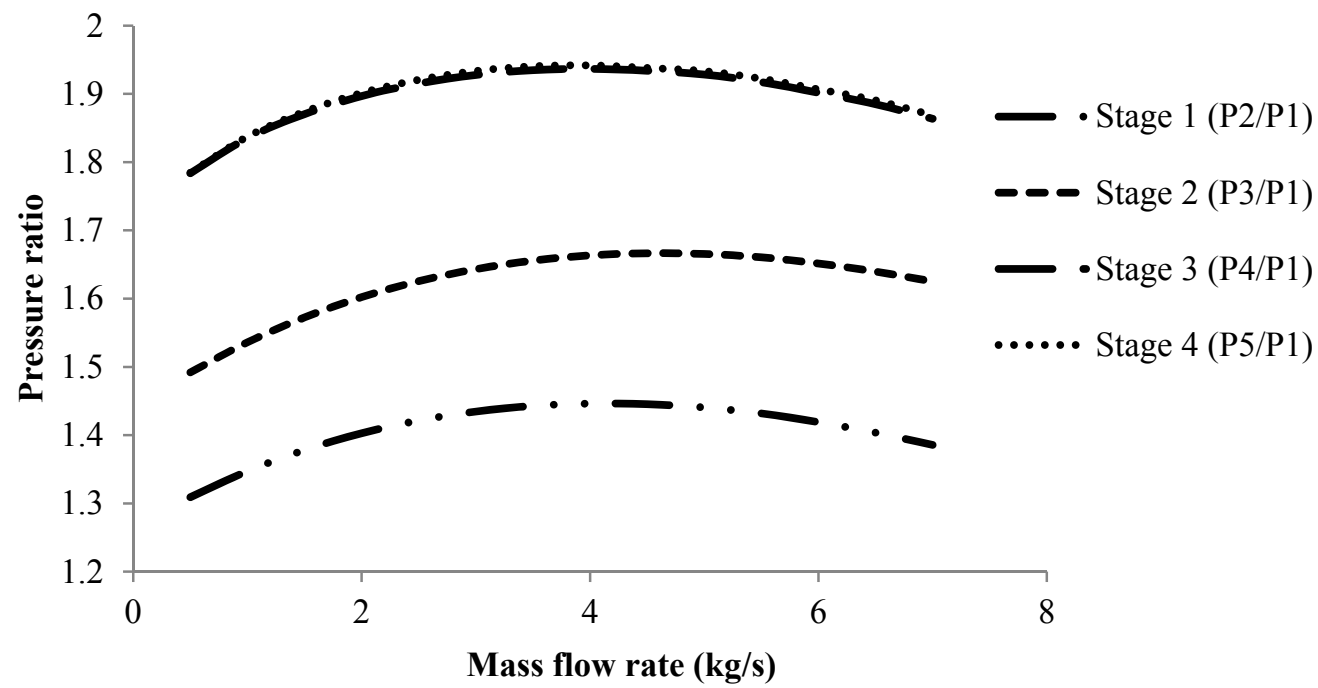

Figure 6. The pressure ratios at each compressor component ( $\mathrm{N}=13000 \mathrm{rpm})$

Figure 6 presents the pressure ratio against mass flow rate characteristics obtained from simulation under rotational speeds at $13,000 \mathrm{rpm}$. It is very helpful to show the process of the pressure ratio building up at different stage inside of the centrifugal compressor. The observations from the figure are listed as follows:

Impeller contributes the most to the pressure building up in all the components, then does vaned diffuser and vaneless diffuse.

It is clear to find that Volute contribute little to the pressure recovery in the whole compressor system, which also proves that the volute is used for directing the air flow rather than improve the compressor performance. 


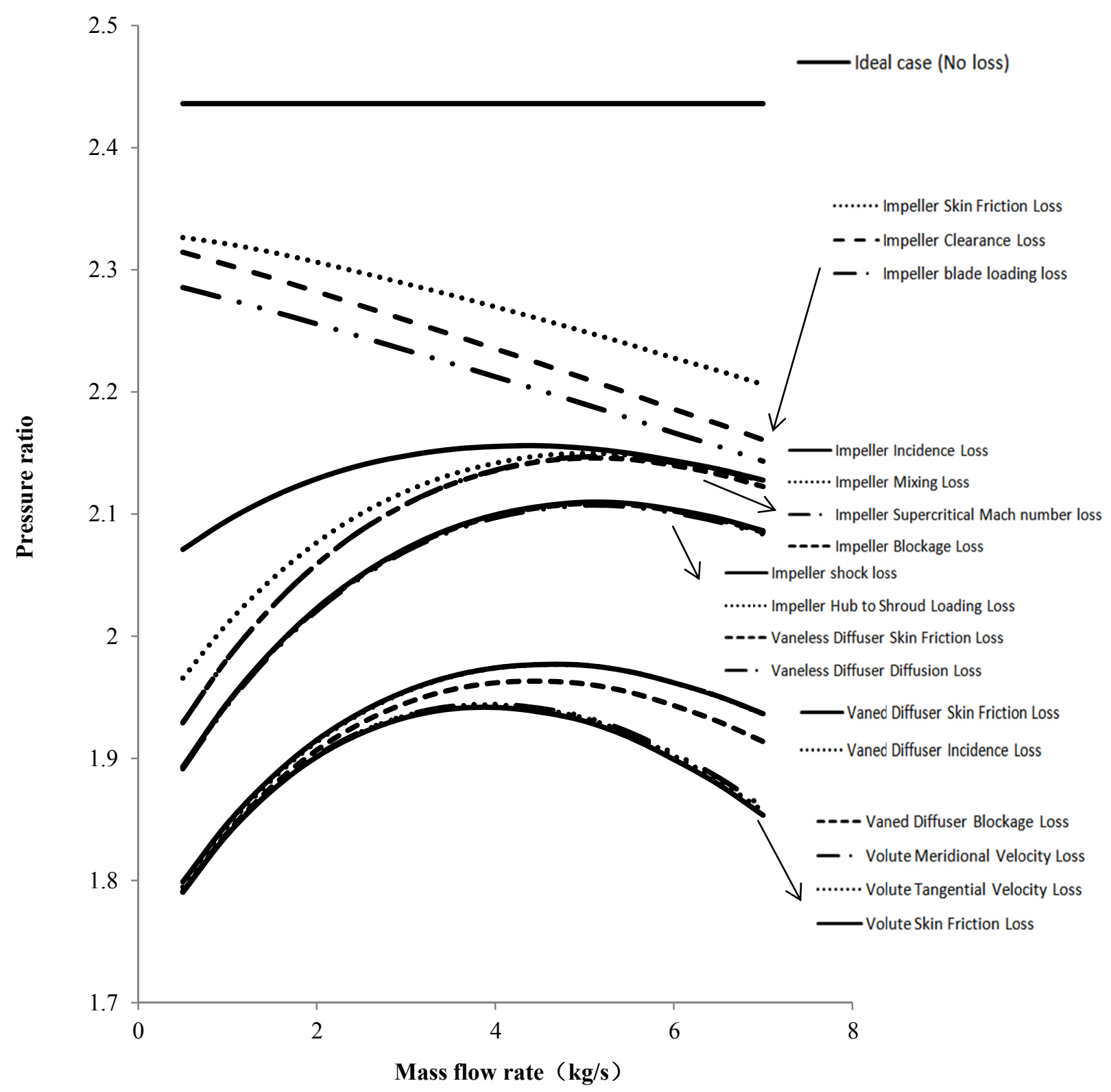

Figure 7. Effects of Different losses on the Compressor Performance (N=13000rpm)

Figure 7 shows the effects of different losses on the compressor performance. The observations from the figure are listed as follows:

In ideal condition, the pressure ratio keeps unchanged at constant rotational speed for different mass flow rate.

From the gaps between each lines in the figure 5, it is easy to find the dominate losses that shape the compressor performance line mostly. The impeller incidence loss, impeller skin friction loss and vaned diffuser skin friction loss are the most important losses in centrifugal compressor.

It also can be seen that impeller incidence loss contribute the most to the shaping of the performance curve.

Another losses such as Impeller clearance loss, impeller blade loading loss, impeller shock loss, vaned diffuser incidence loss and vaned diffuser blockage loss cannot be ignored, although they contribute less than the three dominate losses. 
Other losses contribute little to the shaping of performance curve, such as the losses in the volute. It also demonstrate that the volute contributes little to the pressure recovery in the whole compressor system, the losses in volute affect the compressor performance slightly.

Under the guide of this figure, it is easy to distinguish which losses are pronounced in each component of the centrifugal compressor. The compressor performance can be significantly improved by reducing the main losses in the compressor.

The parametric studies on the main loss mechanisms have been taken to determine the effect of the losses on compressor performance under different operating conditions.

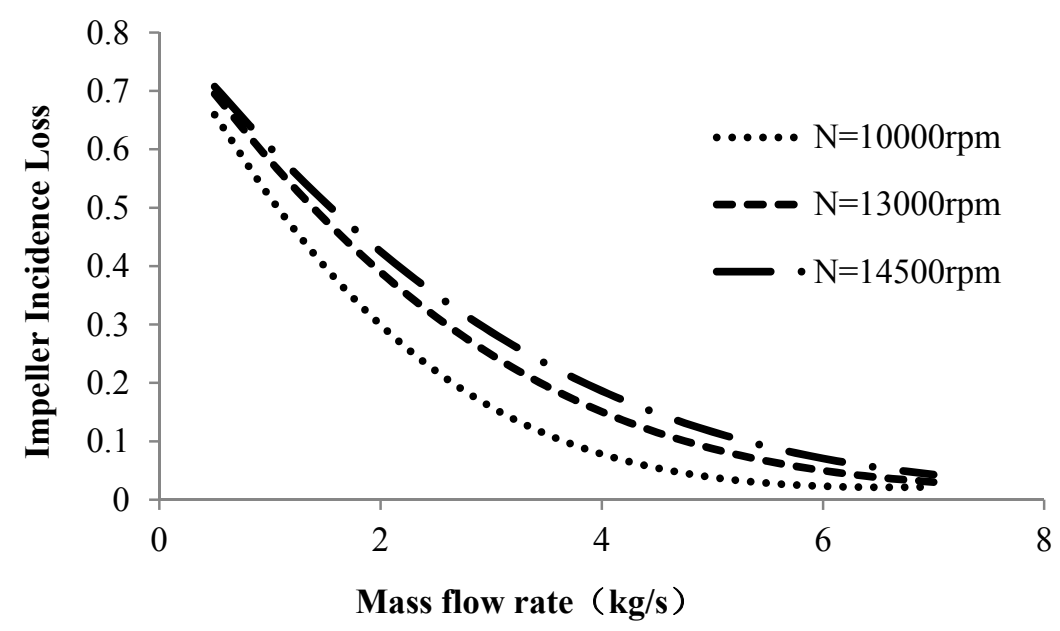

Figure 8. Impeller Incidence loss under different rotational speed

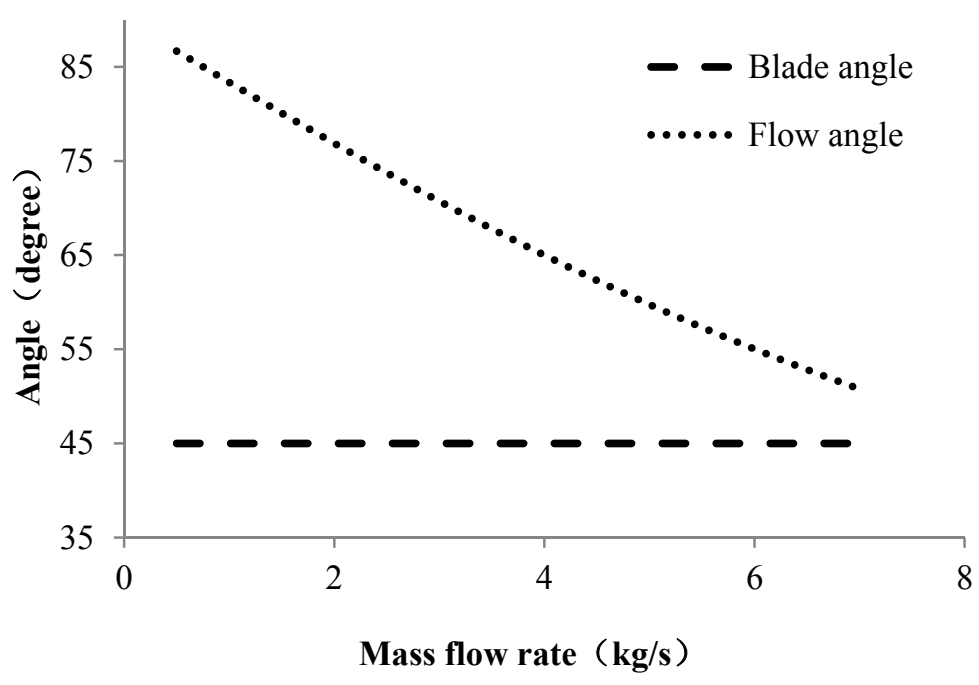

Figure 9. the relation between the flow angle and the mass flow rate

From figure 6, it can be seen that the incidence loss decreases as the absolute incidence angle decreases. The reason for it can be the larger the incidence angle, the larger the required change in flow direction and therefore the larger the loss. Figure 7 shows the relation between the flow angle and the mass flow rate. With the increase of mass flow rate, the flow angle gets closer to the blade angle. The difference between the flow angle and the blade angle is the incidence angle. 


\section{Application}

With the model, it is possible for a parametric study on the effect of each loss mechanism on the performance, and which can aid the designer in justifying design decisions minimising the magnitude of the losses and thus positively influence the overall performance. The compressor model can also be applied as a sub-system to investigate the two stage Turbocharging or Variable Geometry Turbocharger (VGT) in the future.

\section{Conclusion}

In this paper, One-dimensional (1D) compressor model based on the thermodynamics has been constructed for the analysis of compressor performance. This model investigated the effect of each loss on the compressor performance. For the impeller, there are nine losses affecting the performance. They are Shock loss, Incidence loss, Clearance Loss, Skin friction loss, Blade loading loss, Hub to shroud loading loss, Blockage loss, Mixing loss and Supercritical Mach number loss.

There are two loss models employed in the vaneless diffuser sub-model, they are Skin friction loss and Diffusion loss. And for the vaned diffuser sub-model, four loss models are applied. They are Skin friction loss, Incidence loss, Blockage loss and Wake mixing loss.

Finally, there are three loss models equipped in the volute sub-model. They are Meridional velocity loss, Tangential velocity loss and Skin friction loss. With all the loss models, it will guide the designer to identify the most important losses to focus on in order to improve performance at the least cost.

The compressor model is also validated with the experimental results found in public literature. Compare with the results in the public literature, the simulation results here match with the experiment results more accurately, thanks to the various loss models added in. The simulation results also give the stable area compressor operating in by labeling the surge line. However, the simulation results shows a little underestimated with the experiment results when the mass flow rate goes up. The reason for this might be due to some other losses (disc friction, choking, etc.), which are not taken account in this model.

From the analysis of the different losses affecting the compressor performance, it can be seen that the pressure ratio keeps unchanged at constant rotational speed for different mass flow rate if there was no loss at all. For all the losses in centrifugal compressor, Skin friction loss in Impeller and vaned diffuser impact compressor performance mostly. Impeller incidence loss is another dominant loss and it shapes the pressure ratio curve significantly. Since the volute contributes little to the pressure recovery in the whole compressor system, the losses in volute affect the compressor performance slightly.

\section{References}

Aungier, R. H. (1995). Mean streamline aerodynamic performance analysis of centrifugal compressors. Journal of Turbomachinery, 117(3), 360-366. http://dx.doi.org/10.1115/1.2835669

Baines, N. C. (2005). Fundamentals of turbocharging. Concepts NREC.

Chapman, K. S., Keshavarz, A., \& Honegger, U. (2007). Variable Geometry Turbocharger. Pipeline Research Council International, Inc. Report No. PR-266-9920, Virginia.

Dixon, S. L. (2005). Fluid mechanics and thermodynamics of turbomachinery. Elsevier Science.

Herbert, V. (1980). A method of performance prediction for centrifugal compressors. National Gas Turbine Establishment.

Japikse, D., \& Baines, N. C. (1995). Introduction to turbomachinery. Concepts ETI.

Johnston, J. P., \& Dean, R. C. (1965). Losses in vaneless diffusers of centrifugal compressors and pumps: Analysis, experiment, and design. New York, N.Y.: ASME.

Moussa, Z. M. (1978). Impeller Casing Clearance Gap Flow Prediction Program, Carrier Corp. Technical Report 9-1030-05.

Oh, H. W., Yoon, E. S., \& Chung, M. K. (1997). An optimum set of loss models for performance prediction of centrifugal compressors. Proceedings of the Institution of Mechanical Engineers, Part A: Journal of Power and Energy, 211 A4(4), 331.

Takagi, T., Kobayashi, J., Miyashiro, H., \& Morimoto, H. (1980). Performance prediction of single-suction centrifugal pumps of different specific speeds. In ASME Twenty-fifth Annual International Gas Turbine Conference and Twenty-second Annual Fluids Engineering Conference on Performance Prediction of Centrifugal Pumps and Compressors (pp. 227-234). New Orleans, Louisiana, March 1980. 
Thanapandi, P., \& Prasad, R. (1990). Performance prediction and loss analysis of low specific speed submersible pumps. Proceedings of the Institution of Mechanical Engineers, Part A: Journal of Power and Energy, 204(4), 243-252. http://dx.doi.org/10.1243/PIME_PROC_1990_204_033_02

Watson, N., \& Janota, M. S. (1982). Turbocharging the internal combustion engine: Macmillan.

Weber, C. R., \& Koronowski, M. E. (1986). Meanline Performance Predictions of Volutes in Centrifugal Compressors. New York, N.Y.: ASME.

Whitfield, A., \& Baines, N. C. (1990). Design of radial turbomachines. Longman Scientific $\backslash \&$ Technical.

Yahya, S. M. (2005). Turbines compressors and fans. Tata McGraw-Hill.

\section{Copyrights}

Copyright for this article is retained by the author(s), with first publication rights granted to the journal.

This is an open-access article distributed under the terms and conditions of the Creative Commons Attribution license (http://creativecommons.org/licenses/by/3.0/). 